\title{
Hypocellular myelodysplastic syndrome with myelofibrosis in acute myeloid leukemia transformation: A case report
}

\author{
KUI SONG ${ }^{1,2}$, XIAOJUN XU ${ }^{1}$ and $\mathrm{MIN} \mathrm{LI}^{3}$ \\ ${ }^{1}$ Department of Hematology, The Affiliated Zhongshan Hospital, Sun Yat-Sen University, Zhongshan, Guangdong 528403; \\ Departments of ${ }^{2}$ Hematology and ${ }^{3}$ Pharmacy, The First Affiliated Hospital of Jishou University, \\ Jishou, Hunan 416000, P.R. China
}

Received August 22, 2014; Accepted May 7, 2015

DOI: $10.3892 / \mathrm{ol} .2015 .3247$

\begin{abstract}
Primary myelodysplastic syndrome (MDS) with myelofibrosis is a rare hematological disorder that should be classified as a distinct subgroup of MDS. Treatment of MDS with myelofibrosis remains problematic and the prognosis is poor in these patients, particularly following transformation into acute myeloid leukemia (AML). The current study presents the case of a 28-year-old male diagnosed with MDS associated with myelofibrosis, together with hypocellular bone marrow features. Following induction chemotherapy consisting of mitoxantrone and cytarabine, the patient achieved complete remission, but developed severe myelofibrosis. The patient relapsed and the disease transformed into AML 12 months later. However, the extent of the myelofibrosis was markedly alleviated upon administration of a FLAG regimen that consisted of fludarabine, cytarabine and granulocyte colony-stimulating factor during the AML transformation. After one course of the FLAG regimen, the patient achieved a second complete remission. As there was no suitable donor for hematopoietic stem cell transplantation (HSCT), the patient relapsed and succumbed shortly after. In conclusion, MDS with fibrosis is an aggressive disease, but the degree of myelofibrosis may not be associated with the progression of hypocellular MDS, and allogeneic HSCT remains a potentially curative option for affected patients.
\end{abstract}

\section{Introduction}

Multilineage dysplasia and pancytopenia as a consequence of ineffective hematopoiesis are characteristic features of myelodysplastic syndrome (MDS), a clonal hematopoietic stem cell disorder. With an incidence rate of $11-17 \%$ of all MDS

Correspondence to: Dr Min Li, Department of Pharmacy, The First Affiliated Hospital of Jishou University, Shiji Avenue, Jishou, Hunan 416000, P.R. China

E-mail: zs_hematology@163.com

Key words: myelodysplastic syndrome, myelofibrosis, hypocellular, chemotherapy cases, primary MDS associated with myelofibrosis is relatively rare (1). The pathogenesis of the complication of myelofibrosis in MDS remains to be elucidated. Bone marrow hypocellularity has been demonstrated to be less common in MDS with myelofibrosis (12\%) compared with MDS without myelofibrosis (24\%) (2). As a subgroup of MDS, MDS-refractory anemia with excess blasts (MDS-RAEB) is usually rapidly progressive in clinical evolution. When myelofibrosis is associated with MDS-RAEB, patients display a poorer response to chemotherapy and a shortened survival time (3). However, few cases have been reported, particularly in Asia, which has limited the progress of treatment.

Bone marrow fibrosis is variable and typically graded by the density of reticulin and collagen fibers in marrow replacement (4). In primary myelofibrosis, marrow fibrosis is usually progressive and there is increasing evidence that the bone marrow fibrosis grade has prognostic significance (5). An analysis of a large population of patients with primary myelofibrosis identified risk factors that are highly predictive of the transformation to AML at any time during the course of myelofibrosis. Patients with such factors are candidates for more aggressive therapeutic approaches, including allogeneic hematopoietic stem cell transplantation (HSCT), or experimental therapies (6). Decitabine or a FLAG regimen consisting of fludarabine, cytarabine and granulocyte-colony-stimulating factor (G-CSF) has been proven to be effective in the treatment of patients with relapsed and refractory AML, and high-risk MDS $(7,8)$. These results lead to the use of these agents in a relapsed patient with MDS with myelofibrosis who underwent AML transformation, as reported in the present study. The correlation between the extent of myelofibrosis and the progression of MDS is also discussed.

\section{Case report}

A 28-year-old male presented to The Affiliated Zhongshan Hospital, Sun Yat-Sen University (Zhongshan, Guangdong) in December 2008 with malaise that had persisted for 6 months and gingival hemorrhage that had been apparent for 1 month. An initial hematological examination showed normocytic anemia, consisting of a hemoglobin level of $52 \mathrm{~g} / 1$ (normal range, $120-160 \mathrm{~g} / \mathrm{l})$, a white blood cell count of $2.8 \times 10^{9} / 1$ (normal range, 4.0-10.0x $10^{9} / 1$ ) and a platelet count of $46 \times 10^{9} / 1$ 
(normal range, 100-300x10 $/ 1$ ). Serum biochemical analysis was normal on admission and the patient did not exhibit hepatosplenomegaly, as confirmed by physical examination and color echography. Bone marrow aspiration revealed hypocellular marrow with $12 \%$ blasts (normal range, $<5 \%$; Fig. 1). Cytogenetic analysis of bone marrow cells revealed a normal male karyotype of 46,XY. Bone marrow biopsy revealed prominent hypocellular marrow with grade IV reticulin fibrosis (Fig. 2). The patient was consequently diagnosed with MDS-RAEB associated with myelofibrosis and started on combination chemotherapy with $8 \mathrm{mg}$ mitoxantrone on days $1-3$ and $0.1 \mathrm{~g}$ cytarabine on days 1-5 (MA regimen). Following 2 courses of MA chemotherapy, the patient achieved hematological complete remission with grade I reticulin fibrosis. The white blood cell count had decreased to $1.7 \times 10^{9} / 1$ when the patient was ready to received the sixth course of chemotherapy 11 months later. Bone marrow aspiration revealed slightly hypocellular marrow with trilineage dysplasia and $21 \%$ blasts. Furthermore, biopsy showed normocellular marrow with no myelofibrosis. FLAG chemotherapy was administrated as $50 \mathrm{mg}$ fludarabine and $0.3 \mathrm{~g}$ cytarabine on days $1-5$, plus $5 \mu \mathrm{g} / \mathrm{kg}$ subcutaneous G-CSF daily from day 0 until polymorphonuclear neutrophil recovery. The bone marrow aspiration, performed on day 30 at the end of treatment, showed a reduction in the percentage of blast cells (1.5\%). The patient achieved a second complete remission, but then relapsed again and succumbed within the next two months.

\section{Discussion}

Differentiating between MDS and AML is dependent on adequate bone marrow aspirates, which can normally be collected without difficulty unless there is coexisting myelofibrosis. The main criterion for this differentiation is the blast percentage in the bone marrow (9). Myelofibrosis is a relatively rare finding in primary MDS. In general, myelofibrosis appears to occur with cytogenetic abnormalities and confer a poor prognosis, although the recorded survival rates are associated with the French-American-British subtype (showing a longer survival time in patients with refractory anemia and chronic myelomonocytic leukemia compared with those of refractory anemia with an excess of blasts) (10). The bone marrow smears of MDS with myelofibrosis are usually normocellular or hypercellular. However, hypocellular marrow was found in the present case, which was suggestive of a poor prognosis. When the disease relapsed and transformed into AML, the survival time was shortened markedly in clinical evolution, which suggested that conventional treatment would no longer be effective unless allogeneic HSCT was performed.

The treatment of MDS with myelofibrosis remains an unresolved problem. It is the myelofibrosis that makes the treatment more difficult due to the ease of relapse and transformation into AML. Patients with relapsed and transformed disease experience multi-drug resistance, a poor curative effect and high mortality rates (11). The synergy between the drugs in FLAG chemotherapy enhance cytotoxicity, therefore, it is now widely used in refractory and relapsed acute leukemia, and high-risk MDS treatment (12). In the present case, when the disease transformed into AML, the patient was administered the FLAG regimen and achieved remission, and

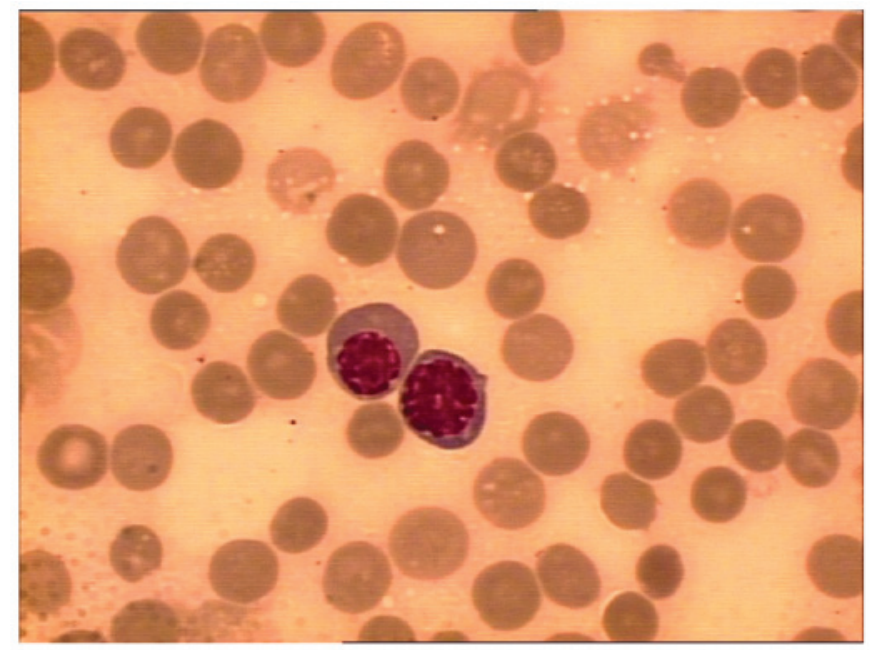

Figure 1. Bone marrow aspirate from the patient showing neutrophil hypogranularity with blasts (Jenner-Giemsa staining; magnification, x1,000).

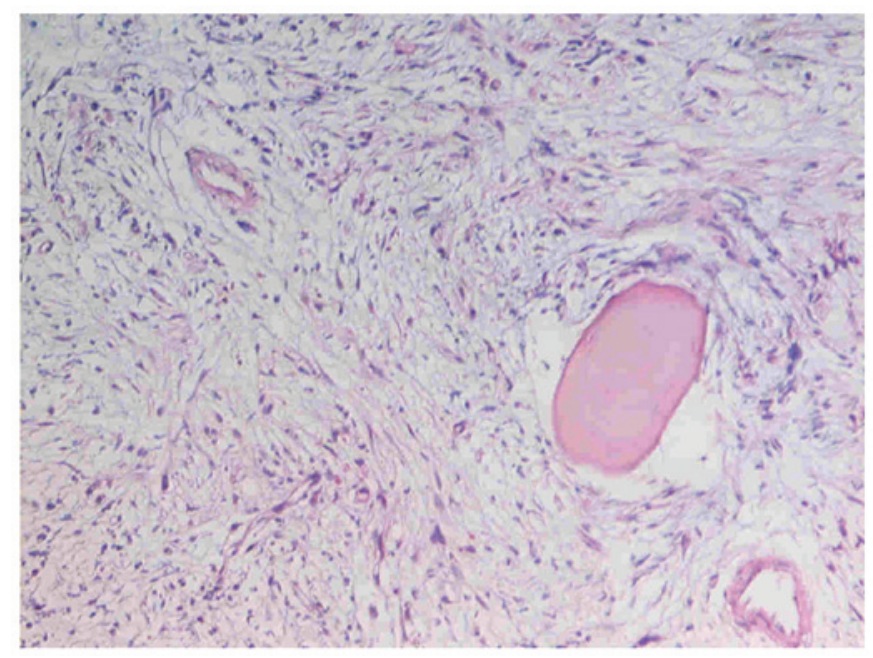

Figure 2. Trephine biopsy of the patient at presentation showing the marked increase in reticulin (hematoxylin and eosin staining; magnification, x200).

the FLAG regimen showed good tolerability. However, the second remission time with FLAG was short, and the induced myelosuppression should also be considered.

The reason why certain MDS patients develop myelofibrosis is not well known. The marrow microenvironment and various cytokines, such as platelet-derived growth factor, calmodulin, transforming growth factor $\beta$ and basic fibroblast growth factor, as well as abnormal megakaryopoiesis, are believed to be significant with regard to the pathogenesis $(2,13)$. From the therapeutical course of the present case, it was indicated that the degree of myelofibrosis maybe not associated with the progress of MDS. A previous study reported that the level of bone marrow reticulin showed little correlation with the severity of the underlying hematological disease (14). Moreover, reticulin fibrosis is often reversible following therapeutic intervention (15). Thus, the improvement of myelofibrosis does not reverse the transformation of MDS to AML.

In the present study, a bone marrow aspiration revealed atypical hypocellular MDS with myelofibrosis. Although MDS with myelofibrosis is associated with the propensity to 
transform into AML and a shortened survival time, the therapeutic response for myelofibrosis maybe not associated with the progression of MDS. Conventional chemotherapy exhibits a limited outcome for MDS patients with myelofibrosis, but allogeneic HSCT or other novel agents may be of use, and require further analysis.

\section{References}

1. Fu B, Ok CY, Goswami M, Xei W, Jaso JM, Muzzafar T, Bueso-Ramos C, Verstovsek S, Garcia-Manero G, Medeiros LJ, et al: The clinical importance of moderate/severe bone marrow fibrosis in patients with therapy-related myelodysplastic syndromes. Ann Hematol 92: 1335-1343, 2013.

2. Akyay A, Olcay L, Kuzu I, Bozdoğan N, Ünal-İnce E, İleri T, Tükün A and Yürür-Kutlay N: A child with myelodysplastic syndrome with hypocellular fibrosis. J Pediatr Hematol Oncol 32: 617-620, 2010.

3. Orazi A and Czader MB: Myelodysplastic syndromes. Am J Clin Pathol 132: 290-305, 2009.

4. Savona MR: Are we altering the natural history of primary myelofibrosis? Leuk Res 38: 1004-1112, 2014.

5. Lekovic D, Gotic M, Perunicic-Jovanovic M, Vidovic A, Bogdanovic A, Jankovic G, Cokic V and Milic N: Contribution of comorbidities and grade of bone marrow fibrosis to the prognosis of survival in patients with primary myelofibrosis. Med Oncol. 31: 869, 2014.

6. Quintás-Cardama A, Kantarjian H, Pierce S, Pierce S, Cortes J and Verstovsek S: Prognostic model to identify patients with myelofibrosis at the highest risk of transformation to acute myeloid leukemia. Clin Lymphoma Myeloma Leuk 13: 315-318, 2013.
7. Saunthararajah Y: Key clinical observations after 5-azacytidine and decitabine treatment of myelodysplastic syndromes suggest practical solutions for better outcomes. Hematology Am Soc Hematol Educ Program 2013: 511-521, 2013.

8. Ossenkoppele GJ, Graveland WJ, Sonneveld P, Daenen SM, Biesma DH, Verdonck LF, Schaafsma MR, Westveer PH, Peters GJ, Noordhuis P, et al: The value of fludarabine in addition to ARA-C and G-CSF in the treatment of patients with high-risk myelodysplastic syndromes and AML in elderly patients. Blood 103: 2908-2913, 2004.

9. Virchis A, Koh M, Rankin P, Mehta A, Potter M, Hoffbrand AV and Prentice HG: Fludarabine, cytosine arabinoside, granulocyte-colony stimulating factor with or without idarubicin in the treatment of high risk acute leukaemia or myelodysplastic syndromes. Br J Haematol 124: 26-32, 2004.

10. Varma $\mathrm{N}$ and Varma S: Proliferative indices, cytogenetics, immunophenotye and other prognostic parameters in myelodysplastic syndromes. Indian J Pathol Microbiol 51: 97-101, 2008.

11. Marisavljević D, Rolović Z, Cemerikić V, Bosković D and Colović M: Myelofibrosis in primary myelodysplastic syndromes: clinical and biological significance. Med Oncol 21: 325-331, 2004.

12. Nokes TJ, Johnson S, Harvey D and Goldstone AH: FLAG is a useful regimen for poor prognosis adult myeloid leukaemias and myelodysplastic syndromes. Leuk Lymphoma 27: 93-101, 1997.

13. Huang WH, Li MS, Chu SC, Wang TF, Kao RH and Wu YF: Thalidomide treatment in a myelofibrosis patient with leukemia transformation. Int J Hematol 99: 188-192, 2014.

14. Della Porta MG and Malcovati L: Myelodysplastic syndromes with bone marrow fibrosis. Haematologica 96: 180-183, 2011.

15. Algarni AA, Akhtari M and Fu K: Myelodysplastic syndrome with myelofibrosis transformed to a precursor B-cell acute lymphoblastic leukemia: A case report with review of the literature. Case Rep Hematol 2012: 207537, 2012. 